parliculièrement ici, n'a plus immédiatement à intervenir.

II est bien évident que des circonstances topographiques spéciales pourront introduire quelques variantes dans ce cadre d'ensemble. Mais si sommaire qu'en soil l'esquisse, elle précise une fois encore des faits essentiels déjà envisagés : d'abord la liaison intime de l'action sylvicole à l'action pastorale, d'où la nécessilé d'une technique sylvo-pastorale plus souple et mieux adaptée que ne pouvail l'être la technique cxclusive du reboisement et des "grands travaux" de consolidation du sol ( $\left.{ }^{1}\right)$; en outre, l'objectif social d'une orientation vers l'enracinement et non l'éviction de la population autochtone qui exploite ces territoires, objectif que ne résoudrait, en aucune manière, l'adoption d'un régime exclusivement pastoral : ce régime sera le corollaire naturel du principe de la protection du sol, mais quand celte dernière aura été assurée. A quoi servirait ce régime pastoräl très vanté, dans de hautes vallées comme celles des Basses-Alpes où, après le succès dı reboisement tel qu'on le poursuit depuis quarante ans, combiné aux autres facteurs d'exode auxquels l'Etat coopère si complaisamment, on sait bien qu'il ne doit plus rester personne. (2)?

TABLEAU IV

\begin{tabular}{|c|c|c|c|}
\hline $\begin{array}{l}\text { ÉLEMENTS } \\
\text { de comparaíon }\end{array}$ & $\begin{array}{c}\text { PAYS } \\
\text { montagneux } \\
\text { du Midi } \\
\text { 31 départements } \\
\text { (1) }\end{array}$ & $\begin{array}{c}\text { SURPLUS } \\
\mathrm{du} \\
\text { territore : } \\
56 \text { départements }\end{array}$ & $\begin{array}{c}\text { TOTALITE } \\
\text { du } \\
\text { territoire } \\
\text { métıopolıtain }\end{array}$ \\
\hline 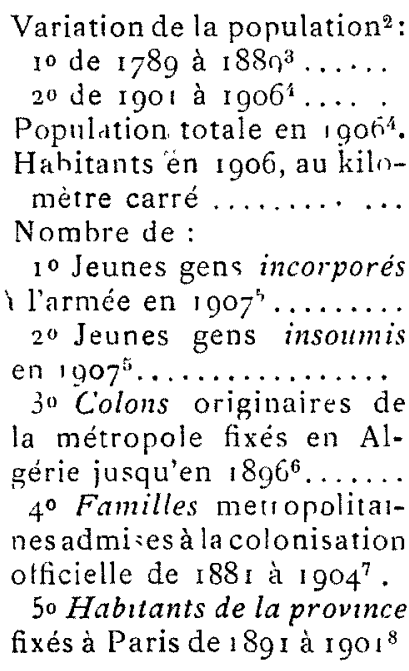 & $\mid \begin{array}{rr}+2726000 \\
63105 \\
10156952 \\
54 \\
72997 \\
1825 \\
62497 \\
5952 \\
63039\end{array}$ & $\begin{array}{r}+\quad 9499000 \\
+\quad 353427 \\
29095315 \\
\\
85\end{array}$ & $\begin{array}{r}+12225000 \\
+\quad 290322 \\
39252267 \\
74 \\
258111 \\
4905\end{array}$ \\
\hline 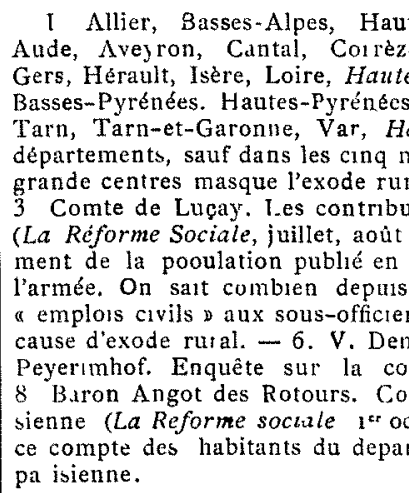 & 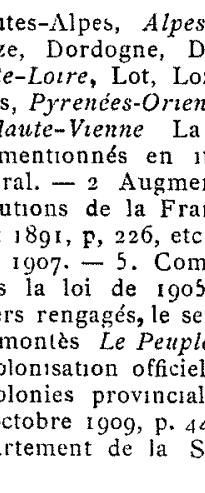 & 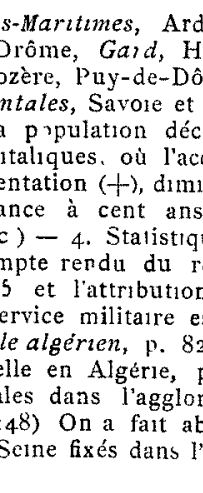 & $\begin{array}{l}\text { dèche, Ariège, } \\
\text { Haute-Garonne, } \\
\text { ôme, Pyrénées, } \\
\text { Haute-Savole. } \\
\text { croît dans ces } \\
\text { ccroissement de } \\
\text { inulion (-) - } \\
\text { s de distance. } \\
\text { que du mouve- } \\
\text { recrutement de } \\
\text { n exclusive des } \\
\text { est devenu une } \\
2 \cdot 84,-7 . \text { De } \\
\text { p. Io8-109. } \\
\text { mération pari- } \\
\text { bstraction dans } \\
\text { l'agglomération }\end{array}$ \\
\hline
\end{tabular}

Quelques brèves indications statistiques nouvelles (Tableau IV) préciscront atilement ces faits contemporains

(1) C'est la technique de l'Ecole nouvelle, Audiffred. Sénat : Séance du 3 mars igro. Compte rendu, p. 382 , col. 2.

(2) Comte A. de Saporta. "Dans les Basses-Alpos ". (Revue des Deux Mondes, $1^{\text {er }}$ juillet rgog. p. 228). d'évasions rurales qui affectent si gravement nos départements montagneux du Midi. Aucunè slatistique ne permet encore d'apprécier la part contributive de la désertion et de l'émigration à l'étranger dans ces causes actuelles de dépopulation $\left({ }^{\mathbf{I}}\right)$.

(A suivre.)

L.-A. FABre, Inspecteur des Eaux et Forêts.

\title{
LÉGISLATION
}

\section{CONSIDÉRATIONS PRATIQUES SUR L'EXPLOITÁTION DES BREVETS D'INVENTION}

\author{
DE LA LICENCE \\ Des effets de la licence (suite)
}

RAPPORTS CONTRACTUELS ENTRE BREVETÉ E'T LICENGIÉ : LEUR SANGTION. - Le contrat de licence fait naître entre les partics contractantes des droits el des obligations. Nous examinerons successivemeñl les obligations du breveté et les obligations du licencié.

\section{Obligations du breveté}

La loi de 1844 ne définil pas le contrat de licence : elle n'en détermine ni les règles, ni les effets. La jurisprudenc: faisant œuvre législative a comblé. les lacunes de la loi et construit la théorie de la licence.

La jurisprudence assimile le contrat de licence au contral de louage. Le breveté qui joue le rôle de bailleur donne au licencié, qui joue le ròle de preneur, le droit de faire usage dé l'invention pour un temps déterminé et sous des conditions déterminées.

L'inventeur breveté qui concède unc licence esl tenu, ell principe, des obligations qui incombent au bailleur d'après. le droit commun. Le licencié qui bénéficie d'une licence est tenu, en principe, des obligations qui pèsent sur le locatairci

La principale obligation assumée par le bailleur est l'obligation de faire jouir le preneur : celte obligation est édictée par les articles r7 r9 el I $_{2}$ I du Code Civil.

Le breveté doit au licencié garantie de la jouissance paisible et garantie des vices de la chose qui en empêchent l'usage.

I. - Garantie de la jouissance paisible. - Le breveté doit assurer au licencić la jouissance paisible de son invention. Le licencié ne peut pas se plaindre tant que sa jouissance n'est pas troublée · un trouble survenant, il peut exercer une action en garantie contre le breveté.

La jouissance du licencié peut être troublée par la jouissance parallèle d'un autre licencié qui tient ses droits du bréveté : le porteur d'une licence simple ne peut pas se plaindre du trouble apporté à sa jouissancc par un autre licencié. Il peut se plaindre si la licence qui lui a été concédée est c: clusive.

Le licencié peut être troublé dans sa jouissance par un. tiers qui ne tient pas ses drnits du breveté, mais qui, bien au contraire, fait échec aux droits du breveté : nous voulon! parler d'un contrefacteur.

(1) Voir notre étude : L' “ Evasion contemporaine des montagnards français ", Annales de la Science Agronomique française et élrangèt: janvier IgI I, p. I à $5 \mathrm{r}$. Nancy, Berger-Levrault. 
Le contrefacteur qui conteste l'existence du monopole du ireveté, trouble sans aucun doute la jouissance du licencié qui est fondé à demander au breveté de faire cesser le trouble, ne pouvant le faire cesser lui-même puisque, en sa qualité de licencié, il n'a pas l'exercice de l'action en contrefaçon.

\section{Plusieurs situations peuvent se présenter.}

$\mathrm{r}^{\mathrm{ro}}$ situation. - Le brevelé poursuil le contrefacteur yui lui a été dénoncé par le licencié et obtient gain de cause.

Le trouble cesse : le breveté a exécuté l'obligation qui lui ncombe. Le licencié n'a pas à se plaindre, tout au plus pourrill-il réclamer au breveté, suivant les circonstances, une indemnité pour le préjudice que lui a causé la contrefaçon.

$2^{8}$ situation. - Le breveté ne poursuit pas le contrefactur qui trouble la jouissance du licencié, malgré une mise en demeure du licencié quii lui signale des faits séricux et incontestables de contrefaçon.

Le breveté manque alors à l'obligalion de garantie dont il est tenu. Le licencié peut demander la résiliation du contrat at des dommages-intérêts pour le préjudice qu'il éprouve d'une concurrence sur laquelle il n'était pas en droil de compter el qui le prive en partie des prolits qu'il aurait réalisés. Jusqu'au jour où sa jouissance a été troublée, lc licencié doit payer les redevances : pour l'avenir le contrat est résilié.

Ce principe a été consacré par un arrêt de la Cour de Paris rendu le if mars rgor dans une affaire W... L...

Par traité du 23 février $1893, W \ldots$ concède à $L$..., pour le département de la Marne, le droit de fabriquer et vendre les muselets de son système breveté moyennant paiement d'une redevance déterminée.

W... et L... stipulent que dans le cas où une contrefaçon se produira, " ils s'entendront pour prendre les mesures nécessaires à la défense de leurs intérêts $"$.

L... est victime de contrefaçons : il avertit W... qui ne poursuit pas. Il le met en demeure, le 16 novembre 1896 , puis l'assigne en résiliation de contrat et en paiement de dommages-intérêts.

Le Tribunal d'Epernay, saisi du litige, prononce la résiliation du contrat de licence, condamne $W$... à payer à $L .$. 1200 francs de dommages-intérêts et déclare que $W \ldots$ a Uroit aux redevances jusqu'au $\mathbf{6} 6$ novembre 1896 , jour de la sommation.

W... fait appel et la Cour de Paris rend, à la date du I 4 mars rgor, l'arrêt suivant.

"Sur la demande de W...,

"Considérant que la réalité et le caractère des faits dénoncés de 1893 à 1896 par L... comme constituant des contrefacons du brevet $W$... ne sont point sérieusement contestés par ce dernier.

"Que L... se trouvant, par suite de ces faits, menacé ou troublé dans la jouissance du brevet dont l'exploitation lui avait été concédée pour le département de la Marne, était en droit de demander à $W$... de faire cesser ce trouble en poursuivant les contrefacteurs.

"Considérant que le traité du 23 février I 893 stipule pour ce cas que les deux parties doivent s'entendre en vue des mesures à prendre pour la défense de leurs droits, il résulte des circonstances et documents visés que L... a fait tout au moins, à la fin de $r 8 \mathrm{~g} 6$, ce qui était en son pouvoir pour que l'entente prévue par la convention se réalisât, qu'il a avisé à plusieurs reprises $W$... de l'existence des contrefaçons, des conditions dans lesquelles elles sétaient produites at de la nécessité de les réprimer.

"Qu'il a, le ro novembre 1896 , par une sommation réguhère, mis W... en demeure d'exécuter le contrat, lui notifiant expressément que, faute d'accomplir son obligation dans un délai déterminé, il cesserait de lui payer les redovances.

"Que W..., malgré cette mise en demeure, n'a lait aucune diligence et n'a pris aucune disposition pour la poursuite des contrelaeteurs.

"Que W... ne saurait, en cel élal des faits, oblenir à son profit la résiliation du traité... qu'elle doil être prononcée ì sa charge exclusive à la dale du ro novembre I 896 .

" Considérant, en ce qui louche les redevances, que si W... a droil au paiement de celles qui seraient dues pour la période antérieure au ro novembre 1896 , il n'esl pas fondé à réclamer pour la période écoulée depuis celte date, sauf son recours s'il échel contre L... daus le cas où, ainsi qu'il l'allegue, ce dernier aurail indüment conlinué à partir de colle date, l'cxploitation du brevet.

"Par ces motils,

"Déclare résilié à compter du ro novembre 1896 le trailé du 23 février r 893 aux torts el griefs de W...

"Met l'appellation à nćant : ordonne que ce dont est appel sortira effet.

"Condamne W... aux dépens."

$3^{\circ}$ situation. - Le brevelé exerce des poursuiles, mais sans succès. Le prétendu contrefacleur dénoncé par le licencié, établit que le brevet est nul ou frappé de déchéance.

Le droit à la garantic est ouvert au profit du licencić qui peut demander, conformément à l'article $\mathbf{y} \mathbf{2}^{\mathrm{x}} \mathrm{du}$ Code Civil la résiliation du contrat avec des dommages-intérêts.

Comme l'existence de contrefaçons permet au licencié d'exercer contre le breveté une action on garantic, breveté et licencié feront bien d'envisager celle éventualité au moment où ils contracteront.

Le breveté peut conférer au licenció le droit de poursuivire les contrefacteurs.

Cette clause insérée dans un contrat de licence est de nature à en modifier le caractère et à transformer la licence cn cession. Le licencié ne pourrait, en tous cas, exercer des poursuites en contrefaçon qu'après avoir fait enregistrer son titre à la préfecture conformément aux disposilions de l'article 20 de la loi de 1844 .

Le breveté peut autoriser le licencié à poursuivre les contrefacteurs en son nom. Le licencic poursuivra à ses risques et périls, profitera des condamnations et des dommages-intérêts qui seront alloués.

Ce mode de procéder comporte des risques pour l'inventeur. Le contrefacteur peut opposer et faire prononcer la nullité du brevet qui sert de base aux poursuites. La décision prononçant la nullité scra opposable l'inventeur qui scra tenu à garantic envers le licencié. Le licencié peut exercer des poursuites abusives motivant des condamnations à des dommages-intérêts dont le recouvrement serait poursuivi contre le breveté figurant en nom dans les poursuites.

Le licencié peut-il demander la nullité ou la déchéance du brevet sur lequel un droit de jouissance lui a été concédé ? Certaines décisions de jurisprudence lui dénient ce droit, 
soutenant que le licencié dont la jouissance n'est pas troublée nē peut, malgré l'existence des causes de nullité, demander la nullité du brevet.

Un arrêt de la Cour de Besançon du i 8 juillet igoo pose le principe suivant :

"Attendu que, sans aucun doute, un brevet frappé de déchéance pcut, tant que celle-ci n'est pas judiciairement prononcée, permettre au licencié de concéder des licences valables : que, sans aucun doute encore, le licencié n'est pas recevable à proposer en justice la nullité ou la déchéance d'un brevet tant qu'il en retire des avantages matériels appréciables, parce qu'il est en quelque sorte associé à l'exploitation de l'invention et que les bénéfices qu'il en retire font que n'ayant pas d'intérêt, il n'a pas d'action ".

Cette décision paraît isolée. Ja jurisprudence se prononce en général en favcur du droit du licencié de poursuivre la nullité ou la déchéance du brevet.

Le licencié a intérêt à fairc proclamer la nullité ou la déchéance du brevet dont il jouit, pour éviter de payer les redevances qui sont la contre-partic de sa jouissance, et pour faire résilier son contral.

D'autre part, l'arlicle 34 de la loi de 1844 pose un principe général.

Mais le licencié qui fait prononcer la nullité ou la déchéance du brevel sur lequel s'exerce son drott de judissance n'est pas fondé à soutenir que le contrat intervenu est sans cause et doit être annulé avec restitution des sommes payées ou des redevances versées.

La jurisprudence décide en pareille circonstance qu'il y a lieu do rechercher dans quelles conditions le licencié a joui du brevet et de déterminer le profit qu'il a réalisé.

II. - Garantie des vices de la chose qui en empêcheint l'usage. - D'après les principes du droit commun en malière de lonage, le bailleur doit mettre le preneur à môtre de jouir de la chose louée ou d'en user, obligation qui n'est pas remplie, si la chose se trouve atteinte de vices qui ne permetten $\iota$ pas de l'employer à l'usage auquel elle est destinée dans la commune intention des parties.

En matière de brevets, les vices de la chose qui ne permettent pas de l'employer à l'usage auquel elle est destinée sont la nullité et la déchéance.

Nous avons donné, en traitant de la cession des brevets, l'énumération des causes de nullité et de déchéance ; rappelons que le brevet nul est censé n'avoir jamais existé et que la nullité rétroagit au jour de la délivrance, alors que la déchéance n'affecte le brevel que du jour où elle a été encourue.

Si le brevet est frappé de nullité ou de déchéance au cours de la jouissance conférée au licencié, le licencié est fondé à invoquer les dispositions de l'article I $_{72} \mathrm{I}$ du Code Civil et à demander garantie.

" Il est dû garantie au preneur pour tous les vices ou défauts de la chose lonée qui en empêchent l'usage quand mème le bailleur ne les aurait pas connus lors du bail.

S'il résulte de ces vices ou défauts quelque perte pour le preneur, le bailleur est tenu de l'indemniser. ")

Le licencié peut-il en pareille hypothèse réclamer le remboursement des sommes qu'il a payées et des redevances qu'il a versées ?

Le droit du licencié esl assim: lable au droit du locataire, r'est un droit de jouissance successive et les redevances sont !nes au breveté, de même que les loyers sont dus au bail- leur proportionnelloment à la duréc de la jouissance. L'ob]j. gation du licencié de payer le prix est corrélative à celle du brevelé de le faire jouir.

En cas de concession de licence portant sur un brevet qui est annulé ou frappé de déchéance, la jurisprudence pose le principe suivant.

Si les parties ont traité de bonne foi, dans l'ignorance du vice qui entachait le brevet, le contrat de licence est résilié pour l'avenir à dater du jour où une décision judiciairc a prononcé la nullité du brevet ou proclamé sa déchéance.

Dans le passé, le contrat a été exécuté, les faits matériels de jouissance ne peuvent pas être supprimés et il y a lieu do faire une distinction.

Ou le licencié a joui sans trouble ni concurrence, parce que les causes de nullité ou de déchéance étaient inconnues des tiers. Dans ce cas, le licencié est tenu de payer les redevances stipulées : il pcut néanmoins obtenir des dommagesintérêts pour le préjudice causé par la réduction imprévuo de la durée de son monopole.

Ou le licencié n'a pas eu la jouissance paisible que doit lui assurer le breveté parce que les causes de nullité ou de déchéance étaient connues des tiers qui se sont livrés à des actes de contrefaçon qui ne pouvaient pas être réprimés. Dans ce cas, le licencié a droit au remboursement des sommeş qu'il a versées, déduction faite des avantages qu'il a retirés de la jouissance du brevet. Il y a compte à faire entre les parties sur des bases que détermincront les juridictions saisies de l'examen du litige, en tenant compte des circonstances spéciales de chaque situation soumise à leur appréciation.

La jurisprudence a appliqué ce principe, soit en matière de déchéance soit en matière de nullité de brevet.

\section{I. - Déchéance de brevet.}

Le ${ }^{\text {er }}$ mars IgoI, $P \ldots$ concède à $\mathrm{H}$... et $\mathrm{C}^{1 e}$ une licenco concernant un brevet pris le 29 janvier rgor. Le titulaire du brevet ne paye pas l'annuité échue en rgo3. La déchéance du brevet est révélée soit au licencié soit aux tiers, seulement en $\mathrm{I}$ go5, époque à laquelle $H$... et $\mathrm{C}^{10}$ réclament à $\mathrm{P}$... la restilution des redevances payées s'élevant à r2 950 francs, et. des dommages-intérêts.

Le Tribunal de la Seine rend, à la date du 16 juillet 1907 , le jugement suivant que confirme la Cour de Paris le 7 janvier $\mathrm{Ig} 9 \mathrm{~g}$ :

“Attendu que le $I^{\text {er }}$ mars IgOI, P... a concédé à $H .$. et $\mathrm{C}^{18}$ une licence d'exploitation sur un brevet pris par lui le 29 janvier Igor pour un procédé...

"Que le minimum des redevances ayant été fixé à $500 \mathrm{fr}$. par mois, les redevances payées par $H .$. et $\mathrm{C}^{\text {1e }}$ se sont élevées pour I 903 , Igoh et une partie de I 905 à I 2.950 francs.

"Que P... n'ayant pas payé l'annuité de rgo3, le brevet est tombé dans le domaine public, le 29 janvier rgo3.

"Que H... et $\mathrm{C}^{\mathrm{ie}}$ assignent $\mathrm{P}$... en déchéance, en restitution des $12950 \mathrm{fr}$, en dommages-intérêts à fixer par étal.

"En ce qui concerne la déchéance :

"Attendu que P... déclare s'en rapporter à justice : que le défaut de paiement de l'annuité du brevet échue en igos entraînant la déchéance, celle-ci doit être prononcée.

"En ce qui concerne la restitution des 12950 francs :

"Attendu que le défaut de paicment de l'annuité échue en i go3 n'a été connu qu'en juin rgo5 non seulement de H... et $\mathrm{C}^{\mathrm{ie}}$, mais encore des contrefacteurs.

"Que jusqu'en rgo5, alors aue la déchéance du brevet $P$... 
n'avait été ni obtenue, ni même demandée, la licence concédée pour l'exploitation de ce brevet n'a pas cessé en fait de produire effet, que s'ils ont versé des redevances s'élerant à I 2950 francs, $\mathrm{H} \ldots$ et $\mathrm{C}^{18}$ ont donc joui jusqu'en $\mathrm{rg} 05$, comme si l'annuité de Igo3 n'avait pas été payée, d'un monopole de fait qui leur a permis de vendre le produit P... dans des conditions aussi avantageuses et de réaliser des bénéfices.

"Qu'à raison de cette jouissance du brevet, on ne saurait ordonner la restitution par $\mathrm{P} . .$. à $\mathrm{H} . .$. et $\mathrm{C}^{18}$ des sommes régulièrement reçues.

: En ce qui concerne les dommages-intérêts :

"Attendu que la licence dú brevet P... a été consentie à II... et $\mathrm{C}^{\text {ye }}$ pour la durée du brevet, c'est-à-dire pour 15 ans, à compter de rgor.

"Qu'ćvidemment le défaut de paiement de l'annuité échue tn 1903 , en laissant tomber le brevet dans le domaine 'public, a causé à $\mathrm{H} \ldots$ el $\mathrm{C}^{18}$ un préjudice à dater de igo5, époque à laquelle cette circonstance a été seulement connue, en leur faisant perdre les bénéfices sur lesquels ils pouvaient compter, qu'il leur causera le même préjudice pour l'avenir, quill leur est dû réparation par P... dont la faute a causé la rupture du contrat.

"Qu'il échet de condamner ce dernier à payer des dommages-intérêts à $\mathrm{H} \ldots$ et $\mathrm{C}^{28}$, mais en tenant compte pour la fixation de ces dommages intérêts de ce que $\mathrm{H}$... et $\mathrm{C}^{\text {to }}$ ne paicnt plus de redevannces à $\mathrm{P}$... depuis $\mathrm{x}$ go5 et de ce que, an outre, il y a eu union de $\mathrm{H} \ldots$ et $\mathrm{C}^{10}$ et $\mathrm{P} \ldots$ avec $J \ldots$ et $\mathrm{V} \ldots$. quils poursuivaient comme contrefacteurs pour l'exploitation commune de leurs brevets, ce qui devait évidemment liminuer pour l'avenir les bénéfices de $\mathrm{H} . .$. et $\mathrm{C}^{\mathrm{ie}}$ sur le brevet $\mathrm{P}$...

"Qu'il faut aussi tenir compte de ce qu'il s'agit d'un objet de mode qui peut cesser complètement d'avoir la vogue.

"Que le Tribunal a les éléments suffisants pour fixer les lommages-intérêts à la somme de 8 ooo francs.

"Par ces motifs,

"Prononce la déchéance du brevet P... du 29 janvier sgor. Dérlare $\mathrm{H} \ldots$ et $\mathrm{C}^{10}$ mal fondés dans lcur demande en isstitution de redevances payées à $P$... s'élevant à I $2950 \mathrm{fr}$. lnur 1903 , rgol et partie de $\mathbf{r} 905$.

"Condamne $\mathrm{P} .$. à payer à $\mathrm{H}$... et $\mathrm{C}^{\mathrm{io}} 8$ ooo francs de uummages-intérêts pour le préjudice qu'il leur a causé. "

it suive).

Amédée Bugand.

Avocat à la Cour d'Appel de Lyon.

\section{ACADÉMIE DES SCIENCES}

\section{PHYSIQUE ET ÉLECTRICITE}

Sur les tubes luminescents au néon. Note de M. Georges Claude, présentée par M. d'Al'sonval, dans la séance du 15 mai 1011.

II. G. Claune signale à l'Académie quelques observations qui unt conduit à un perfectionnement capital des tubes luminescents ull néon, c'est-à-dire à la suppression de tout dispositif de rentrée du gaz luminescent.

On sait que dans les tubes de Moore, comme au surplus dans lo lubes de Crookes, une absorption progressive du gaz employé su produit ef qu'en peu de temps le tube s'éteindrait si une soupape électromagnétique ingénieusement combinée n'avait pour mission de laisser rentrer continuellement du gaz destiné à remplacer celui qui disparaît.
En fait, ses premiers essais lui ont donné de médiocres résultats. Les électrodes, très petites, se volatilisaient rapidement et le tube s'éteignait souvent avant formation complète. Il a agrandi progressivement les électrodes et est arrivé à des cylindres creux de cuivre de $25 \mathrm{~mm}$. de diamètre sur $60 \mathrm{~mm}$. de long pour des tubes de $5 \mathrm{~m}$ de long et $30 \mathrm{~mm}$. de diamètrc, traversés par des courants alternatifs de l'ordre de $0,4 \mathrm{~A}$. Ces tubes s'éteignaient encore après 6 à 8 heures de belle luminescence.

II a remarqué que ces électrodes chauffaient encore beaucoup, presque jusqu'au rouge vif, et qu'un dépôt métallique détachable en écailles se formait sur le verre à leur voisinage. Il a noté par exemple un dépôt de I gr. 6 par électrode pour un fonctionnement de 8 heures. La facile vaporisation des métaux dans ces conditions paraît d'ailleurs, comme le lui a confirmé Sir W. Ramsay, une curieuse caractéristique du néon : en particulier, un tube de Plucker à néon en fonctionnement se platinise en quelques minutes au voisinage des électrodes.

M. Claude a pensé qu'il pouvait y avorr une corrélation entre cette rapide vaporisation du métal et l'extinction rapide du tube.

Il a recueill lo dépòt formé, dont l'aspect est celui du cuivre, mais qui est plus cassant; ot l'a țauté par l'acide nitrique dans un tube à essai renversé, immergé lui-mème dans l'acide nitriquc. On recueille toujours ainsi un résidu gazeux insoluble dans l'acide. Avec le dépốt de I gr. 6 ri-dessus mentionné, il a obtenu $2_{2} \mathrm{~cm}^{3} 5$ de résidu gazeux. Ce résidu, envoyé dans un tube de Plucker vidé, muni d'un petil récipient à charbon, donne, après im. mersion de ce dernier dans l'air liquide, et outre le spectre de l'hydrogène, le spectre du néon très not el celui trìs intense do l'hélium. La mème expérience, répété sur un fragment de a gr. 6 de la partie de l'électrode non volatilisée, ne donne que $o^{\mathrm{cm}^{3}} 3$ de résidu gazeux, el dans ce résidu, traité comme ci-dessus, on distingue faiblement la raie 585,2 du néon et pas du tout les raies de I'hélium. Il a d'ailleurs observé que la raie 585,2 apparaît dáns un tube de Plucker neul par simplo charge de ce tube avec de l'air atmosphérique et immersion du récipient à charbon dans l'air liquide. Ainsi, les gaz rares recueillis ne provenaient pas dos électrodes et lion peut conclure que, conformément aux prévisions et en dépit de l'inaclivité chimique attribuée a ces corps, il y a entraînement et fixation par le métal vaporisé. Ces faits sont à rapprocher de ceux observés par $\mathrm{M}$. Troost au sujet du durcissement rapide des tubes de Plucker à hélium.

Il est remarquable que, dans le résidu gazeux obtenu, l'holium paraît exister en proportion au moins égale à celle du néon, alors que le gaz employé pour la charge du tube était du néon presque exempt d'hélium. Pour expliquer celte prédominance singuliere, on peut admettre que le néon est absorbé en quantité plus grande qu'il ne parait, mais reste sous forme de corps dissous lors du traitement par l'acide nitrique. On peut supposer aussi qu'il y a là un effet sélcctif remarquable pour le peu d'helium contenu dans le néon employé. Il y aurait enfin une troisième hypothèse mais qu'on ne pourrait risquer qu'avec des preuves sérieuses à l'appui, c'est que l'hélium résulterait d'une dissociation du néon. M. Claude n'est pas encore en état d'indiquer laquelle de ces hypothèses est la mieux justiflée.

$\mathrm{Au}$ point de vue pratique, puisque la disparition des gaz rares est une conséquence de la vaporisation des électrodes, on aug. mentera la vie du tube en diminuant cette vaporisation. Or, il" suffit pour cela d'augmenter beaucoup les dimensions des électrodes, ce qui montre qu'il s'agit bien d'une vaporisation et non d'un transport d'ordre électrolytique.

C'est ce qu'il a fait, et les électrodes en cuivre des tules de $35 \mathrm{~m}$ de long et $45 \mathrm{~mm}$ de diamètre qu'il a installés au Grand $\mathrm{Pa}$ lais lors du Salon de Y'Automobile avaient une surface do $3 \mathrm{dm}^{2}$ par ampère. L'un de ces tubes a fonctionné zto heures et l'essai s'est terminé par la ruplure accidentelle du tube. La vaporisation pendant ces 210 heures n'a pas été supérieure à $\mathrm{ig}, 4$ à chaque électrode pour un poids d'électrode de $400 \mathrm{~g}$.

On pouvait craindre que la durée relativement longue obser vée cí-dessus n'ait été due à la charge de néon assez grande correspondant à un tube aussi long. Effectivement, les résultats ont 